\title{
Enhanced oral bioavailability of cyclosporine A by liposomes containing a bile salt
}

This article was published in the following Dove Press journal:

International Journal of Nanomedicine

3 May 2011

Number of times this article has been viewed

\section{Peipei Guan' \\ Yi Lu' \\ Jianping $\mathrm{Q}^{\prime}{ }^{\prime}$ \\ Mengmeng Niu' \\ Ruyue Lian' \\ Fuqiang $\mathrm{Hu}^{2}$ \\ Wei Wu'}

'School of Pharmacy, Fudan University, Shanghai, People's Republic of China;

${ }^{2}$ School of Pharmacy, Zhejiang

University, Hangzhou,

People's Republic of China
Correspondence: WeiWu

School of Pharmacy, Fudan University,

826 Zhangheng Road, Shanghai 201203,

People's Republic of China

$\mathrm{Tel}+862151980002$

Fax +862151980002

Email wuwei@shmu.edu.cn

\begin{abstract}
The main purpose of this study was to evaluate liposomes containing a bile salt, sodium deoxycholate (SDC), as oral drug delivery systems to enhance the oral bioavailability of the poorly water-soluble and poorly permeable drug, cyclosporine A (CyA). Liposomes composed of soybean phosphatidylcholine (SPC) and SDC were prepared by a thin-film dispersion method followed by homogenization. Several properties of the liposomes including particle size, polydispersity index, and entrapment efficiency were characterized. The in vitro release of CyA from these liposomes was less than $5 \%$ at 12 hours as measured by a dynamic dialysis method. The pharmacokinetic results in rats showed improved absorption of CyA in SPC/SDC liposomes, compared with CyA-loaded conventional SPC/cholesterol (Chol) liposomes and microemulsion-based Sandimmune Neoral ${ }^{\circledR}$. The relative oral bioavailability of CyA-loaded SPC/SDC and SPC/Chol liposomes was $120.3 \%$ and 98.6\%, respectively, with Sandimmun Neoral as the reference. The enhanced bioavailability of CyA was probably due to facilitated absorption by the liposomes containing SDC rather than improved release rate.
\end{abstract}

Keywords: liposomes, bile salt, sodium deoxycholate, cyclosporine A, oral bioavailability

\section{Introduction}

Cyclosporine $\mathrm{A}(\mathrm{CyA})$ is a lipophilic cyclic polypeptide composed of 11 amino acids, and has been utilized clinically as a potent immunosuppressant to prevent allograft rejection in various organ transplantations and to treat systemic and local autoimmune disorders. ${ }^{1}$ Despite the great therapeutic interest of CyA, its original oil-based oral formulation (Sandimmune ${ }^{\circledR}$ ) shows high intra- and inter-patient variability and poor bioavailability due to its high molecular weight, rigid cyclic structure, and poor watersolubility. ${ }^{2-4}$ P-glycoprotein mediated efflux from the enterocytes and extensive presystemic metabolism in the gut wall and liver further reduces CyA oral bioavailability. ${ }^{5}$ The current clinically available commercial product of CyA is a microemulsion-based pre-concentrate formulation (Sandiummune Neoral ${ }^{\circledR}$ ), which shows relatively high therapeutic oral bioavailability with reduced variability. In spite of the success of Neoral, CyA attracts continuous attention in the field of oral delivery mainly because it has extremely poor water solubility and poor permeability, and represents one of the most challenging model drugs to test the efficiency of oral delivery systems. ${ }^{6,7}$

In the past decade, various nanoscale drug delivery systems have been investigated to increase the oral bioavailability of CyA. ${ }^{6-10}$ Among these vehicles, liposomes show promising potential due to their absorption-enhancing capability and excellent biocompatibility. The conventional liposome formulation composed of phosphatidylcholine and cholesterol showed oral bioavailability similar to Sandimmune Neoral. ${ }^{6}$ 
Nevertheless, there are problems associated with orally administered liposomes when they are exposed to the harsh environment in the gastrointestinal tract. The $\mathrm{pH}$ gradient, pancreatic lipases, and the bile salts can lead to destabilization of the liposomal vesicles, ${ }^{11}$ among which the key role of the bile salts is the most significant. ${ }^{12}$ In view of this, efforts have been made to improve the stability of conventional liposomes for oral delivery, including polymeric coating ${ }^{13,14}$ and encapsulating liposomes into bulk vehicles. ${ }^{15,16}$ In addition, studies revealed that incorporation of bile salts in the lipid bilayers could stabilize the membrane against the detrimental effects of physiological bile acids in the gastrointestinal (GI) tract. ${ }^{17,18}$ Bile salts stabilized vesicles (bilosomes) have shown promising potential in oral vaccine delivery. ${ }^{17,19,20}$ Bilosomes could provide both protection to the antigen from the hostile environment of GI tract as well as enable transmucosal uptake and subsequent immunization. ${ }^{19}$ Thus, it was indicated that liposomes containing bile salts may act as more stable carriers than conventional liposomes and facilitate the transmembrane transport and absorption of drugs.

In our previous study, liposomes containing a bile salt, sodium deoxycholate, were confirmed to enhance the oral bioavailability of a poorly water-soluble drug fenofibrate by 5.13-fold, ${ }^{21}$ which could be interpreted by multiple mechanisms of facilitated solubilization ${ }^{12,22}$ and facilitated absorption through the M-cell uptake pathway. ${ }^{23}$ However, a 4.4-fold increase in oral bioavailability of the same drug can be observed for fast-dissolving solid dispersion formulation..$^{24}$ It seemed that improving dissolution of the poorly water-soluble drugs like fenofibrate, which are classified as BCS II drugs according to the biopharmaceutics classification system, ${ }^{25}$ was a very efficient way of enhancing the oral bioavailability. The superiority of liposomes containing bile salts has not been confirmed in the previous study using a BCS II model drug. Therefore, we sought to confirm the enhancing effect of this new oral drug carrier, using more challenging BCS IV drugs such as CyA, whose bioavailability cannot be simply enhanced by solubilization mechanisms. ${ }^{26}$ In this study, CyA-loaded liposomes containing a bile salt, sodium deoxycholate, were prepared and evaluated both in vitro and in vivo with the aim to enhance the oral bioavailability of CyA.

\section{Materials and methods}

\section{Materials}

Cyclosporine A (CyA) was purchased from the Pharmaceutical Factory of Sichuan Institute of Antibiotic Industries (Chengdu, China). Soybean phosphatidylcholine (SPC) was supplied by Lipoid (Germany). Sodium deoxycholate (SDC) was purchased from Sinopharm Chemical Reagent Co. Ltd (Shanghai, China). Cholesterol (Chol) was obtained from Shanghai Toshisun Enterprise Co. Ltd (Shanghai, China). Sephadex G-50 was purchased from Pharmacia (Uppsala, Sweden). Ultrapure water was prepared by a Milli-Q purification system (Millipore, Molsheim, France). High performance liquid chromatograpy (HPLC)-grade acetonitrile and tert-butyl methyl ether was supplied by TEDIA (Fairfield, USA). Sandimmune Neoral, a microemulsionbased CyA soft capsule (25 mg) (Novartis Pharma GmbH, Germany) was purchased from a local distributor. All other chemicals were of analytical grade and used as received.

\section{Preparation of liposomes containing sodium deoxycholate}

SPC/SDC liposomes were prepared by an improved thin-film dispersion method. ${ }^{21,27}$ In brief, SPC, SDC, and CyA were dissolved in dichloromethane/methanol $(9 / 1, \mathrm{v} / \mathrm{v})$ in a roundbottom flask. Then the organic solvent was removed using a rotary evaporator (RV 10 digital, IKA Works, German) in a $30^{\circ} \mathrm{C}$ water bath (HB 10 digital, IKA Works, German) under vacuum. The dried lipid films were maintained under reduced pressure for 2 hours to remove traces of the solvent. The lipid film was then hydrated with phosphate buffer $(50 \mathrm{mM}$, $\mathrm{pH}$ 7.4) for 30 minutes at $40^{\circ} \mathrm{C}$ to obtain a crude dispersion of the liposomes. The liposome dispersion was further homogenized by passing through a high-pressure homogenizer (Avestin Em-C3, Ottawa, Canada) to obtain an opalescent fine dispersion. The liposome dispersion was stored at $4{ }^{\circ} \mathrm{C}$ until use. For the preparation of SPC/Chol liposomes, similar procedures were followed using $\mathrm{Chol}$ in lieu of SDC.

\section{Particle size and zeta potential measurement}

The particle size of the prepared CyA-loaded liposomes was determined by the dynamic light scattering method using NICOMPTM 380 ZLS Zeta Potential/Particle Sizer (NICOMP Particle Sizing Systems, Santa Barbara, CA). Raw data were collected over 5 minutes at $23^{\circ} \mathrm{C}$ and at an angle of $90^{\circ}$, which were further processed with the ZPW388 software program. The particle size was expressed with volume-weighted Gaussian distribution (with Chi-squared value $<3$ ). ${ }^{28}$ The zeta potential of liposomes was also measured using the same equipment.

\section{Entrapment efficiency}

The entrapment efficiency of CyA in the liposomes was determined by a gel permeation chromatography method. ${ }^{6}$ 
Briefly, the Sephadex G-50 gel-filled column $(20 \mathrm{~cm} \times 1.0 \mathrm{~cm})$ was first eluted with $100 \mu \mathrm{L}$ blank liposomes to saturate the column and improve the column recovery. Subsequently, CyA-loaded SPC/SDC or SPC/Chol liposomes were mounted and eluted with phosphate buffer $(\mathrm{pH} 7.4)$ at a flow rate of $0.5 \mathrm{~mL} /$ minute to separate free CyA from liposomes. The elution was monitored through turbidity measurement at a wavelength of $500 \mathrm{~nm}$ by using a UV-2401 spectrophotometer (Shimadzu, Japan). The eluentcontaining liposomes were dissolved with methanol and analyzed for CyA. The entrapment efficiency (EE\%) was calculated as:

$$
E E \%=W_{c} / W_{t} \times 100 \%
$$

where $W_{\mathrm{c}}$ and $W_{\mathrm{t}}$ denoted the weight of CyA in liposomes and total CyA weight in the liposome dispersion.

\section{Determination of CyA by HPLC}

In this study, CyA in the formulations and release media was determined by an HPLC/UV method. ${ }^{29}$ The LC-10 ATvp HPLC system (Shimadzu, Japan) was composed of a binary pump, a tunable ultraviolet detector, a column heater, and a manual injector. The mobile phase was a mixture of acetonitrile/water/tert-butyl methyl ether/ phosphoric acid $(60 / 35 / 5 / 0.1, \mathrm{v} / \mathrm{v} / \mathrm{v} / \mathrm{v})$, pumped at a flow rate of $1.5 \mathrm{~mL} /$ minute. CyA was separated using a $\mathrm{C} 18$ column (Venusil XBP, $5 \mu \mathrm{m}, 4.6 \mathrm{~mm} \times 150 \mathrm{~mm}$; Agela, Tianjin, China) guarded with a refillable precolumn (C18, $2.0 \mathrm{~mm} \times 20 \mathrm{~mm}$; Alltech, Lexington, USA) and a stainless pre-heating tube (internal diameter: $0.25 \mathrm{~mm}$; length: $1000 \mathrm{~mm}$ ). The detection wavelength and column temperature were set to $226 \mathrm{~nm}$ and $70^{\circ} \mathrm{C}$, respectively. The linearity range of the calibration curve was within $0.515-51.5 \mu \mathrm{g} / \mathrm{mL}$ with a correlation coefficient of 0.9999 .

\section{Transmission electron microscopy}

The morphology of CyA-loaded SPC/SDC liposomes was observed under a high-resolution transmission electron microscopy (TEM) (Tecnai $\mathrm{G}^{2}$ F20, FEI, Holland) with a field-emission gun operating at $200 \mathrm{kV}$ after negative staining. Briefly, liposomes were diluted with phosphate buffer and adsorbed onto a copper grid and air-dried for 1 minute at room temperature after removing the excessive sample with filter paper. A drop of $2 \%$ phosphotungstic acid (w/v, pH 6.5 in distilled water) was then added and the liposomes were stained for 30 seconds. At the end, the sample was air-dried for another 10 minutes at room temperature before TEM observation.

\section{In vitro release study}

In vitro release of CyA from SPC/SDC, SPC/Chol liposomes and Neoral was evaluated by a dynamic dialysis method ${ }^{29}$ in a ZRS-8G release tester (Tianjin, China) according to the Chinese Pharmacopoeia Method III (the small beaker method). The fundamental properties of both SPC/SDC and SPC/Chol liposomes are given in Table 1. Before release test, $3 \mathrm{~mL}$ of $\mathrm{SPC} / \mathrm{SDC}$ and SPC/Chol liposome dispersion were sealed in dialysis bags (MWCO 14,000, Millipore, Boston, PA), whereas Neoral capsules were dispersed in $12.5 \mathrm{~mL}$ ultrapure water, $3 \mathrm{~mL}$ of which was sealed in dialysis bags. Then the dialysis bags were immersed in $100 \mathrm{~mL}$ release medium containing $0.2 \%(\mathrm{w} / \mathrm{v})$ sodium lauryl sulfate thermostatically maintained at $37 \pm 0.5^{\circ} \mathrm{C}$ and stirred at a rotating speed of $100 \mathrm{rpm}$. At time intervals of $0.5,1,1.5,2,3,4,6,10$, and 12 hours, $0.2 \mathrm{~mL}$ of release sample was withdrawn and centrifuged at 10,000 rpm for 10 minutes at $25^{\circ} \mathrm{C}$. The CyA content was then determined by HPLC as described above.

\section{Sample preparation and determination of CyA in rat whole blood}

CyA in rat whole blood was determined using the HPLC-UV method as described above with modifications. The same LC-10 ATvp HPLC system and Venusil XBP C18 column with guard column and pre-heating assemblies were used as described above, except that the volume ratio of the mobile phase acetonitrile/water/tert-butyl methyl ether/phosphoric acid was changed to 525/425/50/1 (v/v/v/v), and the detection wavelength was set to $210 \mathrm{~nm}$.

To prepare the rat blood samples, CyA was extracted by the reported liquid-liquid extraction procedures with minor

Table I Formulation details of SPC/SDC and SPC/Chol liposomes for in vitro release and in vivo bioavailability studies $(\mathrm{n}=3$ )

\begin{tabular}{llllllll}
\hline $\begin{array}{l}\text { Liposome } \\
\text { type }\end{array}$ & $\begin{array}{l}\text { SPC/SDC } \\
\text { (Chol) ratio }\end{array}$ & $\begin{array}{l}\text { Theoretical SPC } \\
\text { concentration } \\
(\%, \mathbf{w} / \mathbf{)}\end{array}$ & $\begin{array}{l}\text { Theoretical } \\
\text { CyA loading } \\
(\mathbf{m g} / \mathbf{m L})\end{array}$ & $\begin{array}{l}\text { Particle } \\
\text { size }(\mathbf{n m})\end{array}$ & Polydispersity & $\begin{array}{l}\text { Zeta potential } \\
(\mathbf{m V})\end{array}$ & $\begin{array}{l}\text { Entrapment } \\
\text { efficiency }(\%)\end{array}$ \\
\hline SPC/SDC & $3 / 1$ & 5 & 2 & $85.6 \pm 1.0$ & $0.064 \pm 0.013$ & $-35.25 \pm 4.42$ & $94.05 \pm 2.76$ \\
SPC/Chol & $5 / 1$ & 5 & 2 & $98.1 \pm 2.3$ & $0.117 \pm 0.032$ & $-32.09 \pm 3.82$ & $95.22 \pm 3.02$ \\
\hline
\end{tabular}

Abbreviations: Chol, cholesterol; CyA, cyclosporine A; SPC, soybean phosphatidylcholine; SDC, sodium deoxycholate. 
modification. $^{28}$ To $300 \mu \mathrm{L}$ rat blood, add $15 \mathrm{mg}$ of sodium fluoride followed by $40 \mu \mathrm{L}$ of internal standard solution (cyclosporine D, $20 \mu \mathrm{g} / \mathrm{mL}$ in methanol). After vortex mixing for 30 seconds, $750 \mu \mathrm{L}$ diethyl ether anhydrous was added and vortex mixed for 7 minutes. After centrifuging at $12,000 \mathrm{rpm}$ for 10 minutes at $4{ }^{\circ} \mathrm{C}$, the organic layer was transferred to another tube and evaporated under a light stream of nitrogen at $37^{\circ} \mathrm{C}$. The residue was reconstituted using $40 \mu \mathrm{L}$ methanol $/ 0.05 \mathrm{~mol} / \mathrm{L}$ hydrochloric acid solution $(6 / 4, v / v)$. After washing the injection solution with $150 \mu \mathrm{L} n$-hexane for 2.5 minutes, and a brief centrifugation at $12000 \mathrm{rpm}$ for 10 minutes at $4^{\circ} \mathrm{C}, 20 \mu \mathrm{L}$ of the solution was injected for HPLC analysis. Quantification was based on the peak area ratio $R\left(A_{\mathrm{CyA}} / A_{\mathrm{CyD}}\right)$. Linearity was observed over the concentration range of $0.147-14.7 \mu \mathrm{g} / \mathrm{mL}$ with correlation coefficients of over 0.99 (a typical calibration curve: $R=0.4036 \mathrm{C}-0.0423, r^{2}=0.9983, \mathrm{n}=7$ ). The lower limit of quantification for the determination of CyA in rat blood was found to be $0.07 \mu \mathrm{g} / \mathrm{mL}$. Accuracy for the determination of CyA in rat blood $(n=9)$ was $93.24 \% \pm 4.16 \%$. Intra-day and inter-day precisions were all below $7 \%$ and the extraction recovery of CyA in rat blood $(n=9)$ was $73.25 \% \pm 5.17 \%$.

\section{Bioavailability study}

The pharmacokinetics of the CyA-loaded SPC/SDC liposomes (Table 1) were compared with those of CyA-loaded SPC/Chol liposomes and Neoral capsules (25 mg/capsule, Sandimmune Neoral) in rats after an oral dose of $15 \mathrm{mg} / \mathrm{kg}$. Male Wistar rats, weighing $280 \pm 20 \mathrm{~g}$, were supplied by the Laboratory Animal Service Center of Fudan University. The rats were housed in an air-conditioned room with controlled temperature $\left(24 \pm 1^{\circ} \mathrm{C}\right)$ and humidity $(55 \% \pm 5 \%)$ and free access to water, and maintained on a 12 hours dark/light cycle. The experiment adhered to the Guidelines of the Ethical Committee of Fudan University on use of experimental animals and followed the principles outlined in the Declaration of Helsinki for all human or animal experimental investigations. All the animals used in the study were fasted overnight before dosing. Animals were randomly assigned into 3 groups with 6 animals in each group. The liposome formulations were administered directly by gavage using 18-gauge oral feeding needle, while the content of Neoral capsules was administrated similarly, but after diluting with $10 \mathrm{~mL}$ ultra pure water per capsule. After administration, about $350 \mu \mathrm{L}$ of the blood was collected through the tail vein into heparinized tubes at time intervals of $0.5,1.0,1.5,2.0$, 2.5, 3.0, 4.0, 6.0, 8.0, 12.0, and 24.0 hours. Blood samples were stored at $-20^{\circ} \mathrm{C}$ until analysis.
Pharmacokinetic analysis was performed by a model-independent method, using the DAS 2.1.1 computer program (issued by the State Food and Drug Administration of China for pharmacokinetic study). $\mathrm{C}_{\max }$ and $\mathrm{T}_{\max }$ were observed as raw data. Area under the curve to the last measurable concentration $\left(\mathrm{AUC}_{0-\mathrm{t}}\right.$ was calculated by the linear trapezoidal rule. Area under the curve extrapolated to infinity $\left(\mathrm{AUC}_{0-\infty}\right)$ was calculated as $\mathrm{AUC}_{0-\mathrm{t}}+C_{\mathrm{t}} / k$, where $C_{\mathrm{t}}$ and $k$ were the last measurable concentration and the elimination constant, respectively.

\section{Statistical analysis}

Raw data were analyzed using SPSS statistical software (version 11.0, SPSS, Inc.). Post hoc multiple comparisons were done using one-way ANOVA. The difference between groups was considered statistically significant with a $P$ value less than 0.05 .

\section{Results and discussion Preparation and characterization of SPC/SDC liposomes}

Liposomes containing sodium deoxycholate can be prepared successfully by a thin-film dispersion/homogenization method with a wide range of particle size and entrapment efficiency. Although we previously reported similar liposomes encapsulating another poorly water-soluble drug fenofibrate, ${ }^{21}$ our preparation was modified when encapsulating CyA in this study. Specifically, we used a dichloromethane/methanol mixture solvent rather than diethyl ether anhydrous in order to dissolve SPC, SDC, and CyA simultaneously because SDC is freely soluble in methanol, but not in dichloromethane or diethyl ether anhydrous. On the other hand, we used phosphate buffer (50 mM, pH 7.4) in this study rather than SDC solution as hydration medium in order to avoid possible formation of micelles or vesicles by cholate itself during hydration.

Since particle size exerts significant influence on the performance of liposomes both in vitro and in vivo, ${ }^{30}$ we first studied the effects of several factors on the particle size and distribution. As shown in Figure 1A and 1C, SPC/SDC liposomes before homogenization was typically 1 micron in size and exhibited wide size distribution. The particle size and polydispersity index (PI) was dramatically reduced after homogenization, and the lowest diameter (around $80 \mathrm{~nm}$ ) of liposomes could be achieved when the homogenization pressure increased to 100 bar (Figure 1B, 1C). Although the particle size dropped dramatically, there was no worry about phasing-out of CyA. Due to its lipophilic nature and high 
A

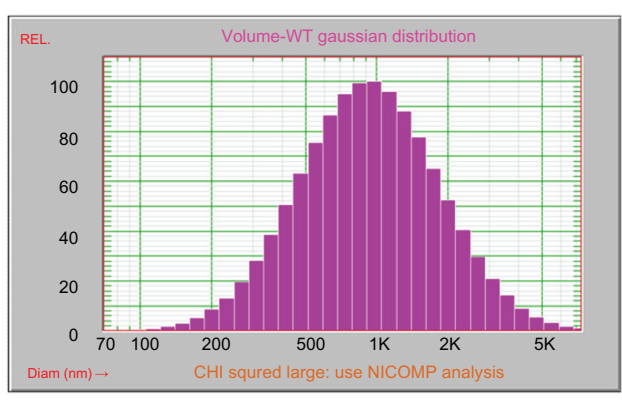

C

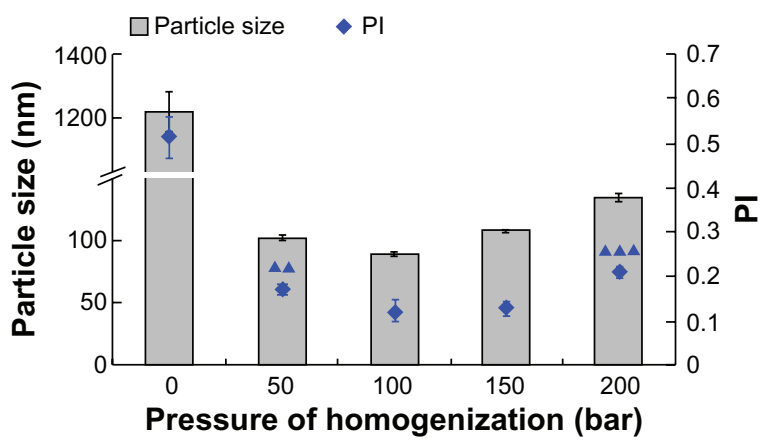

B

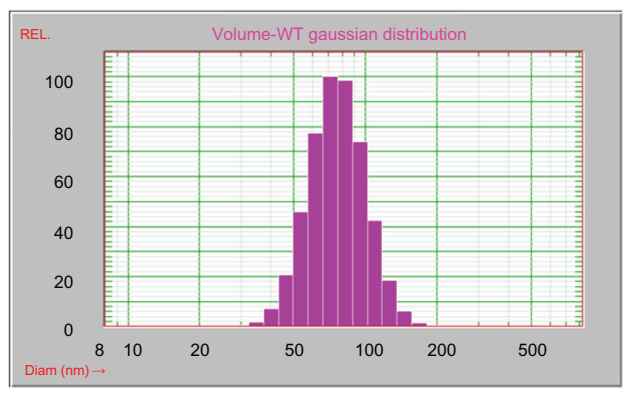

D

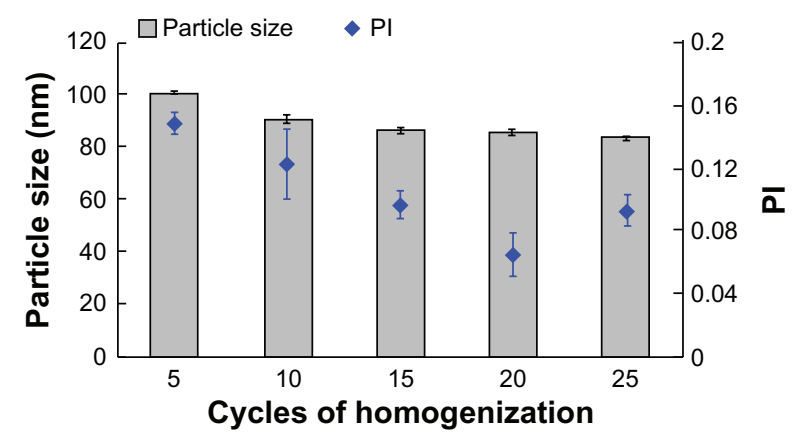

Figure I The effects of homogenization factors on particle size and PI of CyA-loaded SPC/SDC liposomes. Typical size distribution of CyA-loaded SPC/SDC liposomes before (A) or after (B) homogenization is shown. Particle size and PI with different factors including homogenization pressure ( $\mathbf{\Delta} \mathbf{\Delta}<0.0 \mathrm{I}$ comparing $\mathrm{PI}$ at I00 and I50 bar; $\Delta \Delta \Delta P<0.00$ I comparing PI at 100 and I50 bar) $(\mathbf{C})$ and homogenization cycles (D) are shown. Data are presented as mean \pm SD $(n=3)$.

Abbreviations: Chol, cholesterol; CyA, cyclosporine A; PI, polydispersity index; SPC, soybean phosphatidylcholine; SDC, sodium deoxycholate.

affinity with the lipid bilayer, CyA would readily associate with the lipid and maintain a "solubilized" state. Both the TEM observation and entrapment efficiency measurement shown below confirmed the itnegrity of the liposomal structure. However, the particle size and PI were significantly increased $(P<0.01)$ when we further increased the homogenization pressure to $200 \mathrm{bar}$. Rupture and aggregation of the vesicle under large shearing force of homogenization could be employed to interpret this phenomenon. In addition, no significant variation in particle size could be observed at homogenization cycles of more than 5 and the homogenization pressure at $100 \mathrm{bar}(P>0.05)$ (Figure 1D). Nevertheless, the lowest PI could be achieved when we increase the cycles of homogenization to 20 , and rupture and aggregation might occur after 25 times of homogenization, which resulted in increased PI (Figure 1D). Thus we chose a homogenization pressure of either 100 bar or 200 bar to prepare SPC/ SDC liposomes for the following investigation. We chose 300 bar and 20 cycles to homogenize SPC/Chol liposomes, however, which were less flexible, so as to obtain similar mean particle size as SPC/SDC liposomes. Moreover, the micron-scale liposomes might have a multilamellar structure that is common for liposomes produced by the thin-film dispersion method. Further homogenization possibly produced unilamellar liposomes due to the typically small particle size of less than $100 \mathrm{~nm} \cdot{ }^{31}$ Although energy input increased with the increase of homogenization pressure and the number of homogenization cycles, the particle size could not be reduced further because of the significantly increased surface tension. Further reduction in particle size may possibly be achieved through monitoring the composition of the lipids. We did not use extremely small sized particles, which would not favor uptake by the intestinal lymphatic system. ${ }^{32}$

The ratio of SPC/SDC, concentration of SPC and CyA loading also had significant influence on particle size and distribution. A substantial increase in particle size correlated with decrease of SDC in the bilayer with about $80 \mathrm{~nm}$ particle size and PI of 0.1 at a SPC/SDC ratio of 3/1, and there was no significant difference until we increased the SPC/SDC ratio to $4 / 1(P>0.05)$ (Figure $2 \mathrm{~A})$. In contrast, particle size and PI markedly increased when the SPC/ SDC ratio was further increased to $6 / 1$ or $9 / 1$ (Figure 2A). The reduction in particle size and PI as a function of SDC content in lipid bilayers might be attributable to increased flexibility and reduced surface tension of the vesicles. ${ }^{21}$ Our findings were similar to the results obtained by Chingunpitak et al, ${ }^{33}$ namely, that an increase in weight ratio of SDC would reduce the particle size of the suspension 
A

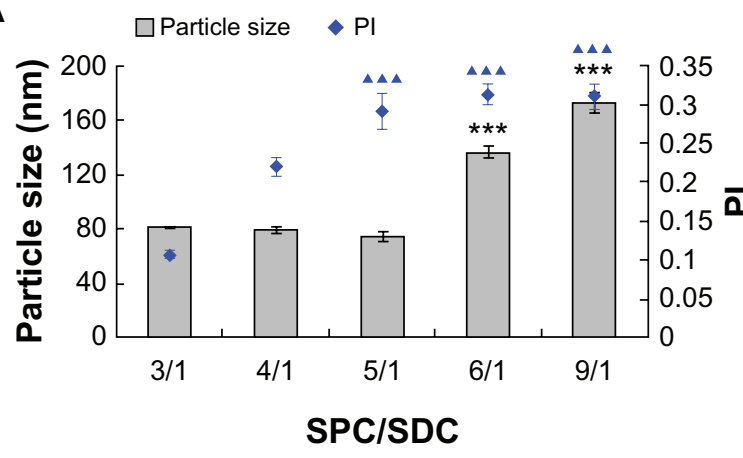

C

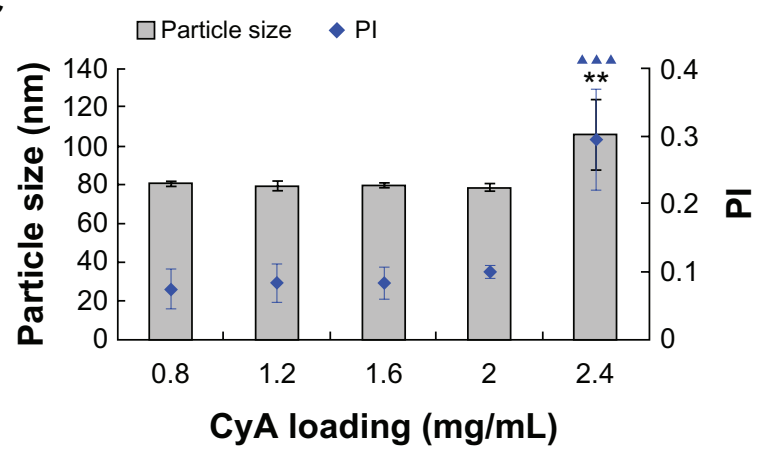

B

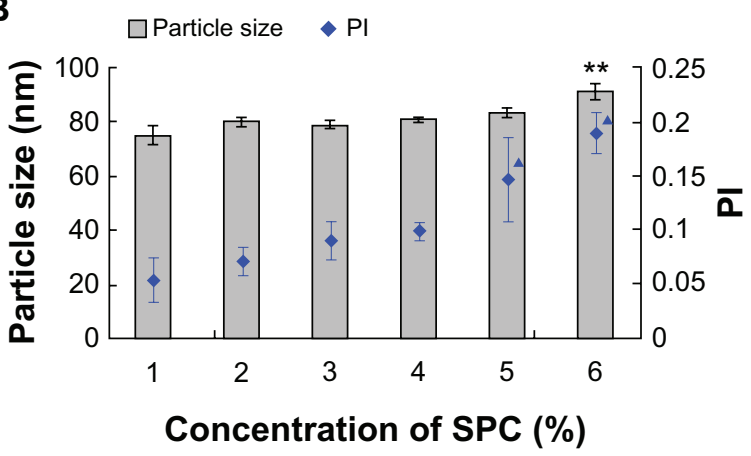

Figure 2 Effect of formulation variables on the particle size and PI of CyA-loaded SPC/SDC liposomes. Ratio of SPC/SDC (A) (***P $<0.00 \mathrm{I}$ comparing particle size in each of the two groups; ***P $<0.00$ I comparing PI at the ratio of $3 / \mathrm{I}$ and $4 / \mathrm{I})$; concentration of SPC (B) (**P $<0.0$ I comparing particle size in each of the two groups; ${ }^{\mathbf{A}} P<0.05$ comparing $\mathrm{PI}$ in each of the two groups.); CyA loading $(\mathbf{C})(* * P<0.0 \mathrm{I}$ comparing particle size in each of the two groups; $\Delta \Delta \mathbf{\Delta} P<0.00 \mathrm{I}$ comparing $\mathrm{PI}$ in each of the two groups). Data are presented as mean $\pm S D(n=3)$.

Abbreviations: Chol, cholesterol; CyA, cyclosporine A; PI, polydispersity index; SPC, soybean phosphatidylcholine; SDC, sodium deoxycholate.

obtained from binary ground mixtures of DHA/SDC. The effect of SPC concentration on particle size and PI was investigated further, and the results showed that the particle size and PI were not significantly influenced by increasing the weight ratio of SPC to $4 \%(P>0.05)$ (Figure $2 \mathrm{~B})$. The liposomes were slightly enlarged, however, with increased PI when the weight ratio of SPC reached $6 \%$, which was possibly due to increased viscosity because of the higher concentration of SPC (Figure 2B). The drug loading had no significant effects $(P>0.05)$ on particle size and PI when the concentration of CyA was below $2 \mathrm{mg} / \mathrm{mL}$. Particle size and PI were dramatically increased at CyA loading of $2.4 \mathrm{mg} / \mathrm{mL}$, however (Figure 2C). Although the exact mechanisms still need further elucidation, possible interpretation includes recrystallization of non-encapsulated drug and significantly increased surface tension.

Figure 3 shows the effect of SPC/SDC ratio, the concentration of SPC and CyA loading on the entrapment efficiency of CyA into SPC/SDC liposomes. Being highly lipophilic, CyA could be easily encapsulated in the liposomes with high entrapment efficiency as reported in a previous study. ${ }^{6}$ However, the results still indicated that the entrapment efficiency of CyA was slightly reduced with decreased content of SDC in the liposomal bilayers (Figure 3A). Similar results were also reported by Sun et $\mathrm{al}^{34}$ showing that SDC could improve the entrapment efficiency of hexamethylmelamine, a lipophilic drug, although this process was phosphatidylcholine-dependent. It was also reported that SDC, with surface-active properties, can incorporate perpendicularly into the surface of the bilayer membrane, perturbate the acyl chains of the lipid matrix, increase the flexibility of the membrane, and thereby improve the solubility of highly lipophilic drug in the membrane. ${ }^{35}$ When the ratio of SPC/ SDC was greater than $4 / 1$, however, the change in entrapment efficiency was not significant, suggesting that the capacity of solubilization by SDC was limited, a result similar to that reported by Sun et al. ${ }^{34} \mathrm{~A}$ significant increase in entrapment efficiency was observed with the increase of SPC content at the weight ratio of $3 \%$ (Figure 3B), which may be attributable to the fact that higher lipid content offers more lipophilic space for CyA. When the lipid content was increased further from $3 \%$ to $6 \%$, however, the amount of CyA loading in liposomes showed no marked change. Further experiments showed that entrapment efficiency was not affected by drug loading when the drug concentration was below $2 \mathrm{mg} / \mathrm{mL}$, whereas the entrapment efficiency significantly 
A

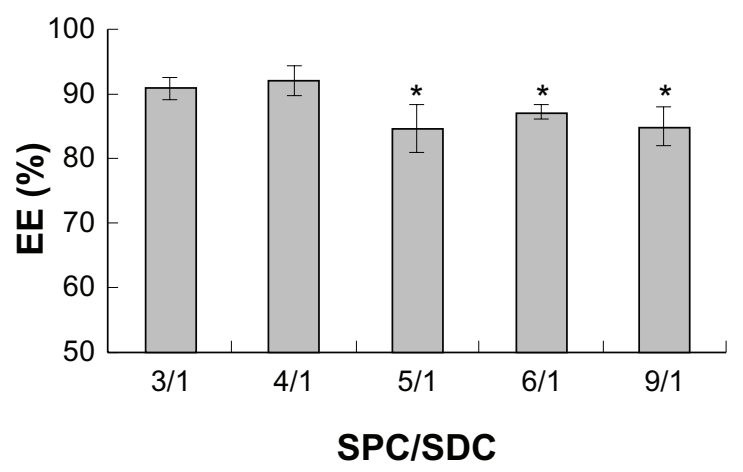

C

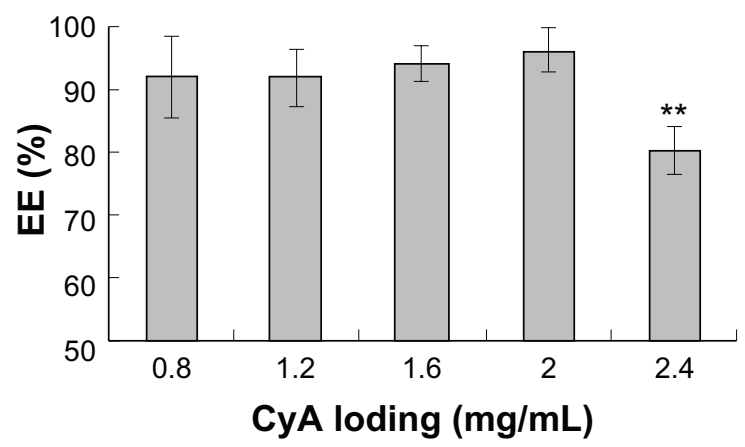

B

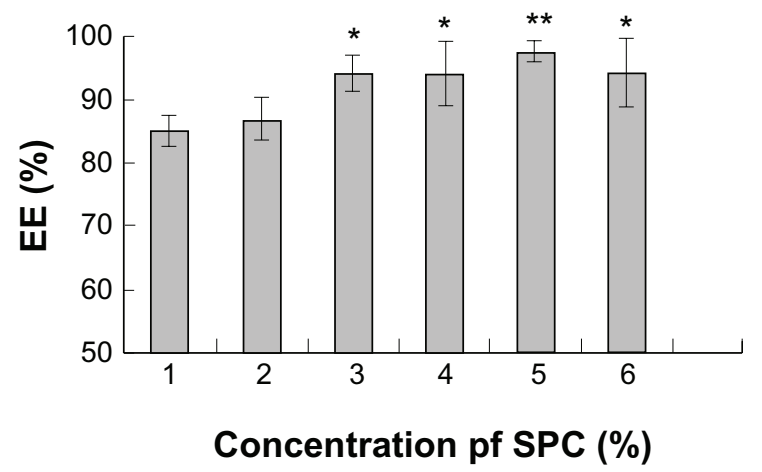

Figure 3 Effect of formulation variables on the entrapment efficiency of CyA-loaded SPC/SDC liposomes. SPC/Chol (A) (*P $<0.05$ comparing SPC/Chol ratio 3/I and 4/I); concentration of SPC (B) ( $* P<0.05$ comparing SPC concentration I\% and $2 \%$; **P $<0.0$ I comparing SPC concentration I\% and $2 \%$ ); CyA loading (C) (**P $<0.0$ I comparing any other group). Data are presented as mean $\pm S D(n=3)$.

Abbreviations: Chol, cholesterol; CyA, cyclosporine A; PI, polydispersity index; SPC, soybean phosphatidylcholine; SDC, sodium deoxycholate.

decreased when CyA was increased to $2.4 \mathrm{mg} / \mathrm{mL}(P<0.01)$ (Figure 3C). This observation was inconsistent with our assumption that there was possible phasing out of nonencapsulated CyA as observed for dramatically increased particle size and PI at CyA $2.4 \mathrm{mg} / \mathrm{mL}$ (Figure 2C). Further evidence of white precipitation at CyA loading up to $2.4 \mathrm{mg} / \mathrm{mL}$ in liposomes after a brief centrifugation at $5000 \mathrm{rpm}$ for $10 \mathrm{~min}$ at $25^{\circ} \mathrm{C}$ confirmed our assumption.

The results of zeta potential measurements are shown in Table 1. There was no significant different between SPC/SDC and SPC/Chol liposomes $(P>0.05)$. It was reported that bilosomes exhibited more stability than conventional vesicles in the GI $\operatorname{tract}^{17}$ which, however, cannot be interpreted by zeta potential-based stabilization mechanism. The possible reason for increased stability of bilosome in the GI tract could be attributed to the defending ability of liposomes containing bile salt against the destructive effect of physiological surfactants. ${ }^{18}$ Furthermore, a short-term stability study of 1 week was carried out to insure the quality of the formulation before oral administration. The entrapment efficiency, particle size, and zeta potential of the SPC/SDC liposomes were determined at $0,1,4$, and 7 days after storage at room temperature. As shown in Table 2, the characteristics of the SPC/SDC liposomes changed significantly.

\section{Transmission electron microscopy}

Figure 4 shows the TEM photograph of CyA-loaded SPC/SDC liposomes. The spherical vesicular structure could be easily

Table 2 The stability of SPC/SDC liposomes for 7 days stored in $4^{\circ} \mathrm{C}(\mathrm{n}=3)$

\begin{tabular}{lllll}
\hline Time (days) & Entrapment efficiency (\%) & Particle size (nm) & Polydispersity & Zeta potential (mV) \\
\hline 0 & $93.27 \pm 3.85$ & $87.2 \pm 2.1$ & $0.074 \pm 0.015$ & $-33.05 \pm 2.79$ \\
1 & $94.72 \pm 2.41$ & $85.3 \pm 1.9$ & $0.071 \pm 0.009$ & $-35.22 \pm 3.42$ \\
4 & $92.53 \pm 3.21$ & $88.9 \pm 2.0$ & $0.080 \pm 0.019$ & $-33.56 \pm 3.75$ \\
7 & $90.38 \pm 4.33$ & $90.2 \pm 3.4$ & $0.085 \pm 0.013$ & $-30.45 \pm 3.69$ \\
\hline
\end{tabular}

Abbreviations: SPC, soybean phosphatidylcholine; SDC, sodium deoxycholate. 


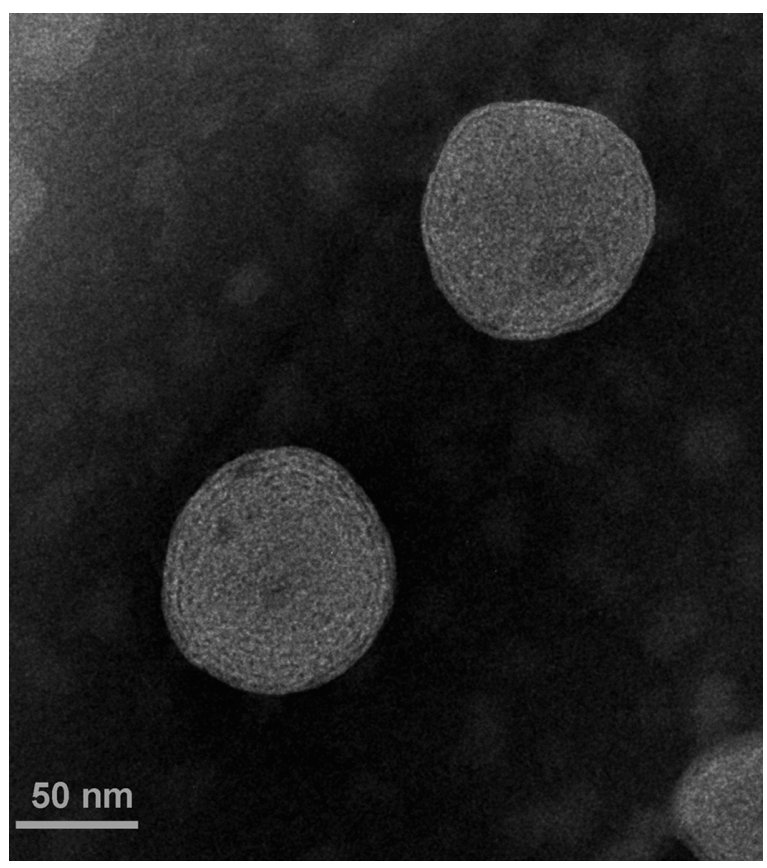

Figure 4 TEM morphology of CyA-loaded SPC/SDC liposomes. Abbreviations: CyA, cyclosporine A; SPC, soybean phosphatidylcholine; SDC, sodium deoxycholate.

identified, which was similar to a previous report of fenofibrateloaded liposomes. ${ }^{21}$ The particle size of CyA-loaded SPC/SDC liposomes was about $90 \mathrm{~nm}$, which was in good correlation with results obtained by particle size measurement.

\section{In vitro release}

Figure 5 shows the in vitro release profiles of the three CyA formulations. The overall release of CyA from CyA-loaded SPC/Chol, SPC/SDC liposomes, and Neoral appeared to be very slow with no more than $5 \%$ at 12 hours. Since the solubility of CyA in $0.2 \%$ SDS solution was about $0.921 \mathrm{mg} / \mathrm{mL},{ }^{29}$ good sink conditions could be maintained

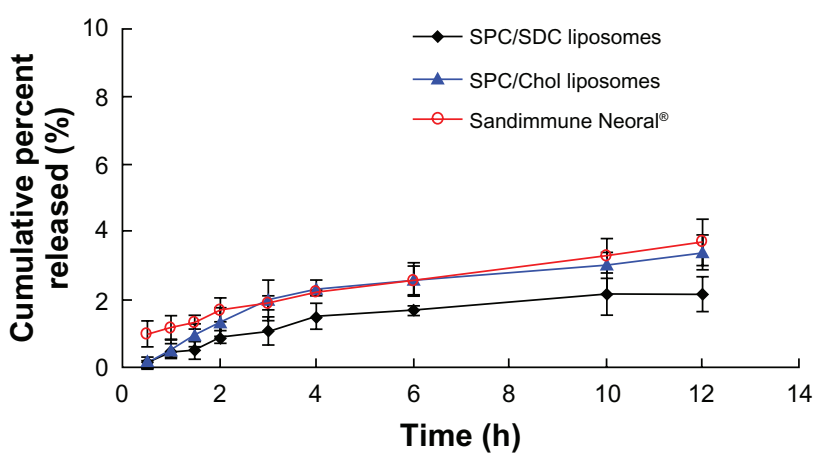

Figure 5 In vitro release of CyA from SPC/SDC, SPC/Chol liposomes, and Sandimmune Neoral. Data are presented as mean \pm SD $(n=3)$.

Abbreviations: Chol, cholesterol; CyA, cyclosporine A; SPC, soybean phosphatidylcholine; SDC, sodium deoxycholate. throughout the release test. CyA in solution could diffuse completely across the dialysis bag within 1 hour, indicating a negligible hindering effect of the dialysis bag on CyA release. Thus the possible reason for slow CyA release from the three formulations was ascribed to the high affinity of CyA with the hydrophobic materials in the formulations. ${ }^{29}$

\section{Pharmacokinetics and oral bioavailability}

To investigate the role of bile salt in enhancing oral absorption of CyA, oral bioavailability of CyA-loaded SPC/SDC liposomes in rats was compared with those of SPC/Chol liposomes and Neoral. The mean whole blood CyA concentration versus time plots of three CyA formulations are shown in Figure 6 and the pharmacokinetic parameters obtained by the statistical moment method are shown in Table 3.

After gavage administration, the $\mathrm{T}_{\max }$ of Neoral was $2.67 \pm 0.69$ hours, which was similar to results reported by Wang et al. ${ }^{36}$ There was no significant difference between the SPC/Chol liposomes and Neoral $(P>0.05)$. However, the $\mathrm{T}_{\max }$ of SPC/SDC liposomes ( $4.67 \pm 1.49$ hours) appeared to be significantly longer than that of both the SPC/Chol liposomes and Neoral $(P<0.01)$. The $\mathrm{C}_{\max }$ of the SPC/SDC liposomes, Neoral, and SPC/Chol liposomes was $2.65 \pm 0.70 \mu \mathrm{g} / \mathrm{mL}$, $2.57 \pm 0.20 \mu \mathrm{g} / \mathrm{mL}$, and $2.28 \pm 0.31 \mu \mathrm{g} / \mathrm{mL}$, respectively, and no significant difference was observed between each of the three formulations $(P>0.05)$. There was also no significant difference in the maximum retention time (MRT) values between either two of the three formulations $(P>0.05)$. SPC/ $\mathrm{SDC}$ liposomes showed significantly higher $\mathrm{AUC}_{0-\mathrm{t}}$ value $(42.16 \pm 6.30 \mu \mathrm{g} \cdot \mathrm{h} / \mathrm{mL})$ than either the SPC/Chol liposomes $(34.56 \pm 4.08 \mu \mathrm{g} \cdot \mathrm{h} / \mathrm{mL})$ or Neoral $(35.02 \pm 4.68 \mu \mathrm{g} \cdot \mathrm{h} / \mathrm{mL})$

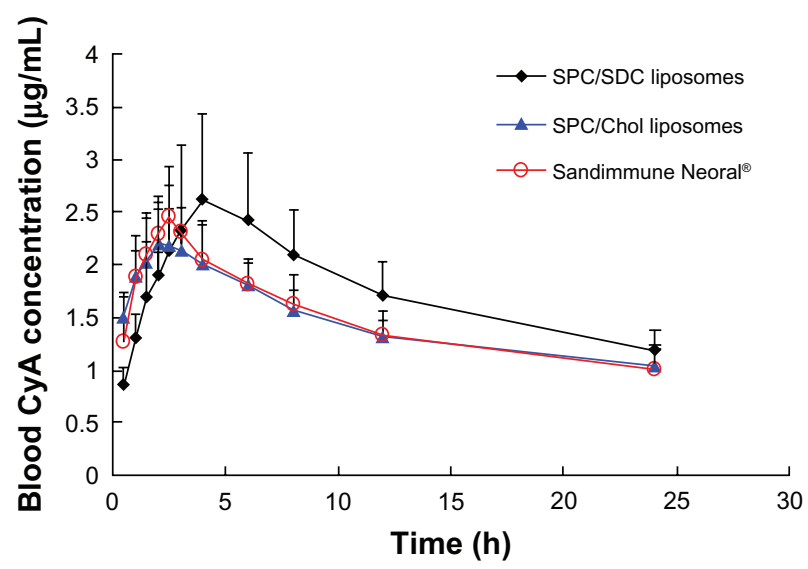

Figure 6 Plot of blood CyA concentration versus time after a single oral dose of $15 \mathrm{mg} / \mathrm{kg}$ equivalent SPC/SDC, SPC/Chol liposomes and Sandimmune Neoral in rats. Data are presented in mean \pm SD $(n=6)$.

Abbreviations: Chol, cholesterol; CyA, cyclosporine A; SPC, soybean phosphatidylcholine; SDC, sodium deoxycholate. 
Table 3 Pharmacokinetic parameters after oral administration of CyA-loaded SPC/SDC, SPC/Chol liposomes and Sandimmune Neoral $(n=6)$

\begin{tabular}{|c|c|c|c|c|c|c|c|}
\hline Formulation & $\mathbf{T}_{\max }(\mathbf{h})$ & $C_{\max }(\mu g / m L)$ & $t_{1 / 2}(h)$ & $\begin{array}{l}\text { AUC }_{0-\mathrm{t}} \\
(\mu \mathrm{g} \cdot \mathrm{h} / \mathrm{mL})\end{array}$ & $\begin{array}{l}A \cup C_{0-\infty} \\
(\mu g \cdot h / m L)\end{array}$ & MRT (h) & $\begin{array}{l}\text { Relative } \\
\text { bioavailability* }\end{array}$ \\
\hline SPC/SDC liposomes & $4.67 \pm 1.49$ & $2.65 \pm 0.70$ & $20.54 \pm 7.63$ & $42.16 \pm 6.30$ & $73.90 \pm 6.63$ & $10.73 \pm 0.90$ & $120.3 \%$ \\
\hline SPC/Chol liposomes & $2.67 \pm 0.69$ & $2.28 \pm 0.31$ & $19.84 \pm 6.50$ & $34.56 \pm 4.08$ & $60.49 \pm 10.79$ & $10.42 \pm 0.37$ & $98.6 \%$ \\
\hline Sandimmun Neoral ${ }^{\circledR}$ & $2.67 \pm 0.69$ & $2.57 \pm 0.20$ & $19.09 \pm 10.15$ & $35.02 \pm 4.68$ & $65.41 \pm 29.55$ & $10.23 \pm 0.64$ & \\
\hline
\end{tabular}

Note: *Relative bioavailability (\%) of the liposomes calculated on $\mathrm{AUC}_{0-\mathrm{t}}$ with Sandimmune Neoral as the reference.

Abbreviations: Chol, cholesterol; CyA, cyclosporine A; soybean phosphatidylcholine; SDC, sodium deoxycholate; MRT, maximum retention time.

$(P<0.05)$. The relative bioavailability, which was calculated on $\mathrm{AUC}_{0 \uparrow \uparrow}$, of the SPC/SDC and SPC/Chol liposomes was $120.3 \%$ and $98.6 \%$, respectively, compared with Neoral. The results indicated that conventional liposomes showed similar pharmacokinetic profiles to Neoral, which was in accordance with the results by Guo et al, ${ }^{6}$ whereas SPC/SDC liposomes showed significantly enhanced oral absorption.

In our previous study on fenofibrate-loaded SPC/SDC liposomes, several mechanisms were proposed to interpret the enhanced oral bioavailability of fenofibrate. ${ }^{21}$ It seems that both enhanced solubilization (facilitated transition from liposomal vesicles to mixed micelles) and enhanced uptake of liposomes as integral vesicles through the M-cell pathway are potential mechanisms. However, the enhanced solubilization mechanism might not function because CyA could not easily permeate across the intestinal epithelia as single CyA molecules. Therefore, the potential explanation for enhanced oral bioavailability of CyA by lipid-based vehicles was that the intact liposomal vesicles may promote the uptake by M-cells in the Peyer's patches and increase the absorption through the lymphatic pathway. Our current study extended the previous investigation ${ }^{6}$ to reveal that SPC/SDC liposomes further enhanced oral bioavailability of CyA compared with that of SPC/Chol liposomes and Neoral. Several mechanisms may contribute to the superiority of SPC/SDC liposomes over conventional liposomes. On one hand, incorporation of bile salts in lipid vesicles could stabilize the membrane against the detrimental effects of physiological bile acids in the GI tract ${ }^{17}$ resulting in more stable SPC/SDC liposomes than SPC/Chol liposomes. The stabilizing effect might extend the resident time of SPC/SDC liposomes in the GI tract, resulting in longer $\mathrm{T}_{\text {max }}$ compared with that of SPC/Chol liposomes or Neoral. It was assumed that more intact liposomes would be available for uptake by the M-cell pathway. On the other hand, permeation of intact liposomes through the intestinal epithelia pathway was another potential mechanism. Owing to the penetration-enhancing effect of sodium deoxycholate, enhanced permeation of liposomes containing SDC across the Caco-2 cell monolayer has been observed..$^{37}$ However, these mechanisms need further elucidation in vivo.

\section{Conclusion}

Liposomes containing SDC could be prepared in high SDC content with SPC/SDC ratio of 3/1 by a thin film dispersion method followed by homogenization. The entrapment efficiency of CyA was as high as $95 \%$ with little escape of CyA during the preparation process. The particle size could be easily tailored by adjusting the homogenization pressure and the number of homogenization cycles. The pharmacokinetic results revealed that liposomes containing sodium deoxycholate were superior to the microemulsion-based formulation (Sandiummune Neoral) and conventional liposomes, in improving the oral bioavailability of CyA. Comparison between SPC/SDC and SPC/Chol liposomes highlights the desirable effect of the bile salt SDC on the oral bioavailability of poor water-soluble and poorly permeable drugs. A mechanism of enhanced solubilization could be excluded owing to the fact of limited release of CyA from either the liposomal or the microemulsion-based formulations. Being absorbed as intact vesicles seems to be a possible interpretation to the enhanced oral bioavailability.

\section{Acknowledgments}

This work was supported by grants from the National Key Basic Research Program of China (2009CB930300), and partly by the Shanghai Municipal Commision of Education (10SG05) and the National Key Innovative Drugs Program of China (2009ZX09310-006, 2009ZX09301-011, 2009ZX09313-008).

\section{Disclosure}

The authors disclose no conflicts of interest.

\section{References}

1. Dunn CJ, Wagstaff AJ, Perry CM, Plosker GL, Goa KL. Cyclosporin: an updated review of the pharmacokinetic properties, clinical efficacy and tolerability of a microemulsion-based formulation $\left(\mathrm{Neoral}^{\mathbb{}}\right)$ in organ transplantation. Drugs. 2001;61(13):1957-2016.

2. Denton MD, Magee CC, Sayegh MH. Immunosuppressive strategies in transplantation. Lancet. 1999;353(9158):1083-1091.

3. Noble S, Markham A. Cyclosporine: a review of the pharmacokinetic properties, clinical efficacy and tolerability of a microemulsion-based formulation $\left(\mathrm{Neoral}^{\circledR}\right.$ ). Drugs. 1995;50(5):924-941. 
4. Czogalla A. Oral cyclosporine A - the current picture of its liposomal and other delivery systems. Cell Mol Biol Lett. 2009;14(1):139-152.

5. Hebert MF. Contributions of hepatic and intestinal metabolism and P-glycoprotein to cyclosporine and tacrolimus oral drug delivery. $A d v$ Drug Deliv Rev. 1997;27(2-3):201-214.

6. Guo JX, Ping QN, Chen Y. Pharmacokinetic behavior of cyclosporin A in rabbits by oral administration of lecithin vesicle and sandimmun neoral. Int J Pharm. 2001;216(1-2):17-21.

7. Müller RH, Runge S, Ravelli V, Mehnert W, Thünemann AF, Souto EB. Oral bioavailability of cyclosporine: Solid lipid nanoparticles (SLN $\left.{ }^{\circledR}\right)$ versus drug nanocrystals. Int J Pharm. 2006;317(1):82-89.

8. Italia JL, Bhatt DK, Bhardwaj V, Tikoo K, Kumar M. PLGA nanoparticles for oral delivery of cyclosporine: Nephrotoxicity and pharmacokinetic studies in comparison to Sandimmune Neoral ${ }^{\circledR}$. J Control Release. 2007;119(2):197-206.

9. Dai JD, Nagai T, Wang XQ, Zhang T, Meng M, Zhang Q. pH-sensitive nanoparticles for improving the oral bioavailability of cyclosporine A. Int J Pharm. 2004;280(1-2):229-240.

10. Wang XQ, Huang J, Dai JD, et al. Long-term studies on the stability and oral bioavailability of cyclosporine A nanoparticle colloid. Int J Pharm. 2006;322(1-2):146-153.

11. Hermida LG, Sabés-Xamaní M, Barnadas-Rodríguez R. Combined strategies for liposome characterization during in vitro digestion. J Liposome Res. 2009;19(3):207-219.

12. Andrieux K, Forte L, Lesieur S, Paternostre M, Ollivon M, Grabielle-Madelmont C. Solubilisation of dipalmitoylphosphatidylcholine bilayers by sodium taurocholate: A model to study the stability of liposomes in the gastrointestinal tract and their mechanism of interaction with a model bile salt. Eur J Pharm Biopharm. 2009;71(2):346-355.

13. Werle M, Takeuchi H. Chitosan-aprotinin coated liposomes for oral peptide delivery: Development, characterisation and in vivo evaluation. Int J Pharm. 2009;370(1-2):26-32.

14. Amin M, Jaafari MR, Tafaghodi M. Impact of chitosan coating of anionic liposomes on clearance rate, mucosal and systemic immune responses following nasal administration in rabbits. Colloid Surface B. 2009;74(1):225-229.

15. Park HJ, Lee CM, Lee YB, Lee KY. Controlled release of cyclosporin A from liposomes-in-microsphere as an oral delivery systems. Biotechnol Bioproc Eng. 2006;11(6):526-529.

16. Lee J-S, Chung D, Lee HG. Preparation and characterization of calcium pectinate gel beads entrapping catechin-loaded liposomes. Int $J$ Biol Macromol. 2008;42(2):178-184.

17. Conacher M, Alexander J, Brewer JM. Oral immunisation with peptide and protein antigens by formulation in lipid vesicles incorporating bile salts (bilosomes). Vaccine. 2001;19(20-22):2965-2974.

18. Senior K. Bilosomes: the answer to oral vaccine delivery? Drug Discov Today. 2001;6(20):1031-1032.

19. Shukla A, Khatri K, Gupta PN, Goyal AK, Mehta A, Vyas SP. Oral immunization against hepatitis B using bile salt stabilized vesicles (bilosomes). J Pharm Pharmaceut Sci. 2008;11(1):58-66.

20. Mann JFS, Ferro VA, Mullen AB, et al. Optimisation of a lipid based oral delivery system containing A/Panama influenza haemagglutinin. Vaccine. 2004;22(19):2425-2429.

21. Chen YP, Lu Y, Chen JM, et al. Enhanced bioavailability of the poorly water-soluble drug fenofibrate by using liposomes containing a bile salt. Int J Pharm. 2009;376(1-2):153-160.
22. Hildebrand A, Beyer K, Neubert R, Garidel P, Blume A. Temperature dependence of the interaction of cholate and deoxycholate with fluid model membranes and their solubilization into mixed micelles. Colloid Surface B. 2003;32(4):335-351.

23. Clark MA, Jepson MA, Hirst BH. Exploiting $M$ cells for drug and vaccine delivery. Adv Drug Deliv Rev. 2001;50(1-2):81-106.

24. Tang N, Lai J, Chen Y, Lu Y, Wu W. Fenofibrate solid dispersion pellets prepared by fluid-bed coating: physical characterization, improved dissolution and oral bioavailability in beagle dogs. J Chin Pharm Sci. 2009; 18:156-161.

25. Yu LX, Amidon GL, Polli JE, et al. Biopharmaceutics classification system: the scientific basis for biowaiver extensions. Pharm Res. 2002; 19(7):921-925.

26. Liu C, Wu JH, Shi B, Zhang YX, Gao TK, Pei YY. Enhancing the bioavailability of cyclosporine a using solid dispersion containing polyoxyethylene (40) stearate. Drug Dev Ind Pharm. 2006;32(1):115-123.

27. Geusens B, Lambert J, De Smedt SC, Buyens K, Sanders NN, Van Gele M. Ultradeformable cationic liposomes for delivery of small interfering RNA (siRNA) into human primary melanocytes. $J$ Control Release. 2009;133(3):214-220.

28. Niu GQ, Cogburn B, Hughes J. Preparation and characterization of doxorubicin liposomes. In: Grobmyer SR, Moudgil BM, editors. Cancer Nanotechnology Methods and Protocols. New York: Humana Press; 2010;624:211-219.

29. Lai J, Lu Y, Yin Z, Hu F, Wu W. Pharmacokinetics and enhanced oral bioavailability in beagle dogs of cyclosporine A encapsulated in glyceryl monooleate/poloxamer 407 cubic nanoparticles. Int J Nanomedicine. 2010;5:13-23.

30. Sun WT, Zou WW, Huang GH, Li AG, Zhang N. Pharmacokinetics and targeting property of TFu-loaded liposomes with different sizes after intravenous and oral administration. J Drug Target. 2008;16(5): 357-365.

31. Yang L, Yang WZ, Bi DZ, Zeng Q. A novel method to prepare highly encapsulated interferon-alpha-2b containing liposomes for intramuscular sustained release. Eur J Pharm Biopharm. 2006;64(1):9-15.

32. Niu M, Lu Y, Hovgaard L, Wu W. Liposomes containing glycocholate as potential oral insulin delivery systems: preparation, in vitro characterization and improved protection against enzymatic degradation. Int J Nanomedicine. 2011;6: in press.

33. Chingunpitak J, Puttipipatkhachorn S, Chavalitshewinkoon-Petmitr P, Tozuka Y, Moribe K, Yamamoto K. Formation, physical stability and in vitro antimalarial activity of dihydroartemisinin nanosuspensions obtained by co-grinding method. Drug Dev Ind Pharm. 2008;34(3):314-322.

34. Sun JK, Deng YG, Wang SL, Cao JN, Gao XF, Dong XD. Liposomes incorporating sodium deoxycholate for hexamethylmelamine (HMM) oral delivery: Development, characterization, and in vivo evaluation. Drug Deliv. 2010;17(3):164-170.

35. Elorza MA, Elorza B, Chantres JR. Stability of liposomal formulations: action of amphiphilic molecules. Int J Pharm. 1997;158(2):173-183.

36. Wang XQ, Dai JD, Chen Z, et al. Bioavailability and pharmacokinetics of cyclosporine A-loaded $\mathrm{pH}$-sensitive nanoparticles for oral administration. J Control Release. 2004;97(3):421-429.

37. Song KH, Chung SJ, Shim CK. Enhanced intestinal absorption of salmon calcitonin ( $\mathrm{sCT}$ ) from proliposomes containing bile salts. J Control Release. 2005;106(3):298-308.
International Journal of Nanomedicine

\section{Publish your work in this journal}

The International Journal of Nanomedicine is an international, peerreviewed journal focusing on the application of nanotechnology in diagnostics, therapeutics, and drug delivery systems throughout the biomedical field. This journal is indexed on PubMed Central, MedLine, CAS, SciSearch $\AA$, Current Contents ${ }^{\circledR} /$ Clinical Medicine,
Dovepress

Journal Citation Reports/Science Edition, EMBase, Scopus and the Elsevier Bibliographic databases. The manuscript management system is completely online and includes a very quick and fair peer-review system, which is all easy to use. Visit http://www.dovepress.com/ testimonials.php to read real quotes from published authors. 